Network Working Group

Request for Comments: 1652

Obsoletes: 1426

Category: Standards Track
J. Klensin, WG Chair

MCI

N. Freed, Editor Innosoft M. Rose

Dover Beach Consulting, Inc.

E. Stefferud

Network Management Associates, Inc.

D. Crocker

Silicon Graphics, Inc. July 1994

\title{
SMTP Service Extension for 8bit-MIMEtransport
}

Status of this Memo

This document specifies an Internet standards track protocol for the Internet community, and requests discussion and suggestions for improvements. Please refer to the current edition of the "Internet Official Protocol Standards" (STD 1) for the standardization state and status of this protocol. Distribution of this memo is unlimited.

Abstract

This memo defines an extension to the SMTP service whereby an SMTP content body consisting of text containing octets outside of the USASCII octet range (hex 00-7F) may be relayed using SMTP.

1. Introduction

Although SMTP is widely and robustly deployed, various extensions have been requested by parts of the Internet community. In particular, a significant portion of the Internet community wishes to exchange messages in which the content body consists of a MIME message [3] containing arbitrary octet-aligned material. This memo uses the mechanism described in [5] to define an extension to the SMTP service whereby such contents may be exchanged. Note that this extension does NOT eliminate the possibility of an SMTP server limiting line length; servers are free to implement this extension but nevertheless set a line length limit no lower than 1000 octets. Given that this restriction still applies, this extension does NOT provide a means for transferring unencoded binary via SMTP. 
2. Framework for the 8bit MIME Transport Extension

The 8bit MIME transport extension is laid out as follows:

(1) the name of the SMTP service extension defined here is 8bit-MIMEtransport;

(2) the EHLO keyword value associated with the extension is 8BITMIME;

(3) no parameter is used with the 8BITMIME EHLO keyword;

(4) one optional parameter using the keyword BODY is added to the MAIL FROM command. The value associated with this parameter is a keyword indicating whether a 7bit message (in strict compliance with [1]) or a MIME message (in strict compliance with [3]) with arbitrary octet content is being sent. The syntax of the value is as follows, using the ABNF notation of [2]:

$$
\text { body-value : := "7BIT" / "8BITMIME" }
$$

(5) no additional SMTP verbs are defined by this extension; and,

(6) the next section specifies how support for the extension affects the behavior of a server and client SMTP.

3. The 8bit-MIMEtransport service extension

When a client SMTP wishes to submit (using the MAIL command) a content body consisting of a MIME message containing arbitrary lines of octet-aligned material, it first issues the EHLO command to the server SMTP. If the server SMTP responds with code 250 to the EHLO command, and the response includes the EHLO keyword value 8BITMIME, then the server SMTP is indicating that it supports the extended MAIL command and will accept MIME messages containing arbitrary octetaligned material.

The extended MAIL command is issued by a client SMTP when it wishes to transmit a content body consisting of a MIME message containing arbitrary lines of octet-aligned material. The syntax for this command is identical to the MAIL command in [1], except that a BODY parameter must appear after the address. Only one BODY parameter may be used in a single MAIL command. 
The complete syntax of this extended command is defined in [5]. The esmtp-keyword is BODY and the syntax for esmtp-value is given by the syntax for body-value shown above.

The value associated with the BODY parameter indicates whether the content body which will be passed using the DATA command consists of a MIME message containing some arbitrary octet-aligned material ("8BITMIME") or is encoded entirely in accordance with [1] ("7BIT").

A server which supports the 8-bit MIME transport service extension shall preserve all bits in each octet passed using the DATA command.

Naturally, the usual SMTP data-stuffing algorithm applies so that a content which contains the five-character sequence of

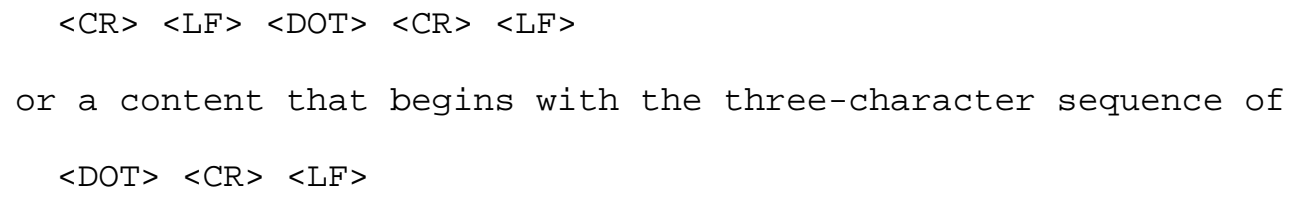


the usual manner for delivery failures. The specifics of the transformation from 8bit MIME to 7bit MIME are not described by this RFC; the conversion is nevertheless constrained in the following ways:

(1) it must cause no loss of information; MIME transport encodings must be employed as needed to insure this is the case, and

(2) the resulting message must be valid 7bit MIME.

4. Usage Example

The following dialogue illustrates the use of the 8bit-MIMEtransport service extension:

S: <wait for connection on TCP port 25>

$\mathrm{C}$ : <open connection to server>

S: 220 dbc.mtview.ca.us SMTP service ready

C: EHLO ymir.claremont.edu

S: 250-dbc.mtview.ca.us says hello

S: 250 8BITMIME

C: MAIL FROM:<nedQymir.claremont.edu> BODY=8BITMIME

S: $250<$ ned@ymir.claremont.edu>... Sender and 8BITMIME ok

C: RCPT TO:<mrose@dbc.mtview.ca.us>

S: $250<$ <roseddbc.mtview.ca.us>... Recipient ok

C: DATA

S: 354 Send 8BITMIME message, ending in CRLF.CRLF. ...

C:

S: 250 OK

C: QUIT

S: 250 Goodbye

5. Security Considerations

This RFC does not discuss security issues and is not believed to raise any security issues not already endemic in electronic mail and present in fully conforming implementations of [1].

6. Acknowledgements

This document represents a synthesis of the ideas of many people and reactions to the ideas and proposals of others. Randall Atkinson, Craig Everhart, Risto Kankkunen, and Greg Vaudreuil contributed ideas and text sufficient to be considered co-authors. Other important suggestions, text, or encouragement came from Harald Alvestrand, Jim Conklin, Mark Crispin, Frank da Cruz, 'Olafur Gudmundsson, Per 
Hedeland, Christian Huitma, Neil Katin, Eliot Lear, Harold A.

Miller, Keith Moore, Dan Oscarsson, Julian Onions, Neil Rickert, John Wagner, Rayan Zachariassen, and the contributions of the entire IETF SMTP Working Group. Of course, none of the individuals are necessarily responsible for the combination of ideas represented here. Indeed, in some cases, the response to a particular criticism was to accept the problem identification but to include an entirely different solution from the one originally proposed.

7. References

[1] Postel, J., "Simple Mail Transfer Protocol", STD 10, RFC 821, USC/Information Sciences Institute, August 1982.

[2] Crocker, D., "Standard for the Format of ARPA Internet Text Messages", STD 11, RFC 822, UDEL, August 1982.

[3] Borenstein, N., and N. Freed, "Multipurpose Internet Mail Extensions", RFC 1521, Bellcore, Innosoft, September 1993.

[4] Moore, K., "Representation of Non-ASCII Text in Internet Message Headers", RFC 1522, University of Tennessee, September 1993.

[5] Klensin, J., Freed, N., Rose, M., Stefferud, E., and D. Crocker, "SMTP Service Extensions", RFC 1651, MCI, Innosoft, Dover Beach Consulting, Inc., Network Management Associates, Inc., Silicon Graphics, Inc., July 1994 .

[6] Partridge, C., "Mail Routing and the Domain System", STD 14, RFC 974, BBN, January 1986.

8. Chair, Editor, and Authors' Addresses

John Klensin, WG Chair

MCI Data Services Division

2100 Reston Parkway, 6th floor

Reston, VA 22091

USA

Phone: : 17037157361

Fax: +1 7037157435

EMail: klensin@mci.net 


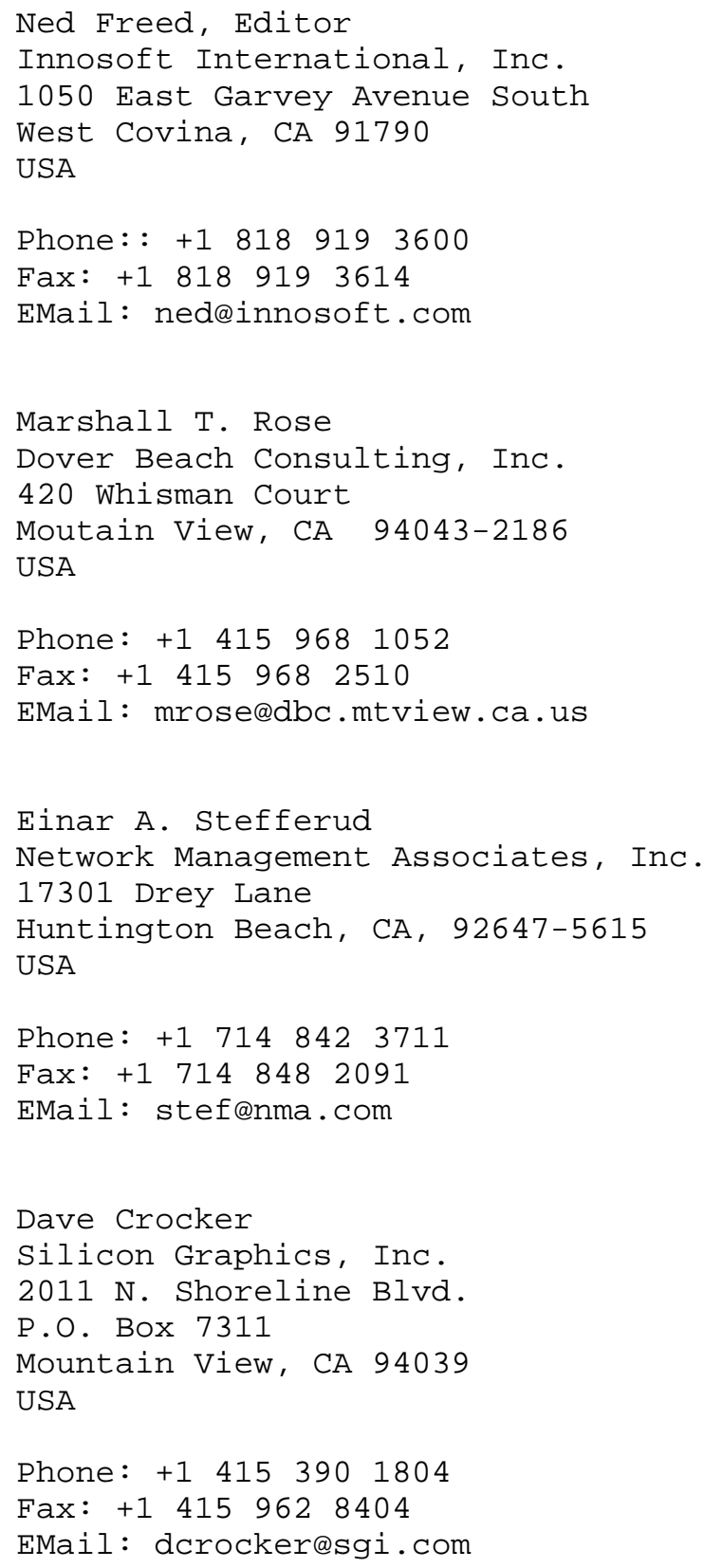

